\section{General practice and public health: fostering collaboration for better health for populations}

\author{
Primary care and public health offer a unique opportunity to support one another, and the important \\ synergies between the two specialties are clear, argue Maggie Rae and Martin Marshall
}

\author{
Maggie Rae, Martin Marshall
}

As the president of the Faculty of Public Health and chair of the Royal College of General Practitioners (RCGP) we know the importance of fostering deep collaboration between primary care and public health. Our members, representing the UK's public health and general practice workforce, face many cross-cutting issues. Both specialties understand that factors rooted in inequality such as homelessness, addiction, poor quality housing, obesity, and smoking all impact profoundly on the health of communities and the individuals that live within them.

General practitioners, given their closeness to the communities they serve, understand the importance of individual and local determinants of health. This role is reflected in the fact that core competencies within the RCGP's training curriculum include "community orientation" and "practising holistically." When consulting with patients, GPs, working with practice staff such as nurses and social prescribers, seek to not only treat illness, but to identify the root causes of ill-health. This insight is invaluable to public health specialists who can then work collaboratively to deliver effective interventions based on clear evidence.

This feedback system between general practice and public health is reciprocal in nature, and just as insight from GPs and practice teams in the community help public health specialists deliver effective interventions at population level, so public health data and principles support GPs in their practice. As well as responding to major incidents and threats to population-level health such as pandemics, public health specialists work with local, regional, and national stakeholders to design, deliver, and evaluate interventions which will protect and improve the health of populations. This work-and the data it generates-supports GPs in enhancing their primary care practice to ensure it is aligned with the needs of the communities they serve.

Public health and general practice staff recognise that to improve health at a local, regional, and national level, we must tackle the underlying issues which impact patients and populations; the wider determinants of health. Both our specialties are concerned with the imperative of reducing health inequalities, with our members seeing the devastating impacts of these disparities, day-in, day-out. And both our specialties understand that to improve health, we must empower communities, offer effective support systems for those in most need, and wherever possible intervene upstream in order to prevent ill-health. General practice and public health staff cannot do this alone, but given the time, resources and tools, many are already demonstrating that there is significant opportunity here.

Public health professionals assess the health needs of the populations they serve, and work with a range of partners to protect and improve health for all. The specialty covers a broad base of work, primarily concerned with three domains of practice; health protection, health improvement, and healthcare public health. All these areas are informed by the evidence and research. Public Health has strong academic specialists which helps strengthen the scientific underpinning of the specialty.

Taken together, these domains of public health form a specialty which can identify the ever-changing needs of populations and deliver effective interventions to improve health outcomes and reduce inequalities. Public health specialists assess current and future health and care needs, so that we can better support communities and individuals.

Protecting the health of populations requires public health professionals to take a broad-based approach to health and wellbeing, delivering work to improve the foundations for good health. This population health approach seeks to reduce inequalities and provide conditions that support good health in our economy, housing, environment, nutrition, transport, planetary health and all other areas that have an impact on health and wellbeing.

Our specialties offer a unique opportunity to support one another, and the important synergies between primary care and public health are clear. There are a range of shining and emerging examples of public health and primary care collaboration in practice. Perhaps one of the most well known is the Bromley by Bow Centre in Tower Hamlets, which serves a population facing severe deprivation, was founded by the community itself in the 1990s. Working with local public health teams, the GP team delivered an exemplary model of primary care which focused not only on treating patients, but addressing the foundational causes of their ill-health. In an area where poor health is driven by deprivation, the centre championed social prescribing to tackle issues of isolation, unemployment, and lack of support. In doing so, GPs could continue to treat their patients, while also working to prevent the root causes of poor health and address the deep inequalities faced by the community.

The interests of our two specialties also converge on issues of policy, especially as our members fight to deliver services following years of austerity. Funding 
cuts and frequent re-organisation of the public health system has limited the capacity of public health teams to build effective working relationships with local general practice. Similarly, GPs have faced workforce shortages and increased clinical pressures which has stifled opportunities for deeper collaboration with public health colleagues.

Properly resourced public health and primary care teams, placed within a framework which allows for effective integration, will work together to deliver improvements in health that our two specialties could not achieve alone. The upcoming developments to the role of Integrated Care Systems perhaps offer an opportunity to foster this deeper collaboration, but serious gaps remain in these plans. What is certain, is that despite the mounting pressures our workforces face in responding to the long term impacts of the covid-19 pandemic, our members will continue to collaborate to protect and improve health for all. 